\section{Social competition in the Mongolian gerbil,} Meriones unguiculatus

STANLEY WECHKIN and ROGER $L$. REID, State University of New York, College at Brockport, Brockport, N.Y. 14420

Gerbils were tested for social dominance under the same and different incentives and under varying levels of motivation. Significant consistency was found within tests and between tests for the same incentive, though none was found between tests for different incentives, nor did consistency vary with level of motivation. These data, along with the absence of any correlated displays, were interpreted as indicating the absence of dominance-regulated competition for this species, such consistency as was found being ascribed to individual differences in the level of skill required for the various tests.

The purpose of the present experiments was to determine the role of dominance relationships in regulating competition among gerbils. Though little is known of the social behavior of gerbils in their natural habitat, and though Thiessen (1968) has suggested that territoriality is characteristic of these animals, in the laboratory gerbils are highly gregarious and engage in no overt hostility once a group has become established. Since gerbils have a formidable aggressive potential, as is evidenced by their frequently lethal attacks upon strange conspecifics, 1 the question arises as to how this aggression is inhibited among familiar animals. In many other aggressive group-living species, dominance-subordination relationships, in which one animal gives way to another on the basis of displays, serve to prevent internecine fighting in competitive situations (Wynne-Edwards, 1962). To be maximally effective, such dominance relationships should be relatively constant over time and serve to regulate competition for more than one kind of incentive. Accordingly, in the first study, gerbils were repeatedly tested in competition both for food and for the avoidance of noxious stimulation, and the consistency of outcomes across these tests was assessed.

\section{EXPERIMENT 1}

\section{Method}

The Ss were 24 adult male gerbils obtained from Western New York Animal Resources, Inc., Ontario, New York, randomly divided into two equal groups and housed four to a cage.
Food competition testing took place in an empty 10-gal aquarium and consisted of simultaneously placing two gerbils, who had been deprived of food for the previous $22 \mathrm{~h}$, with a single small pellet of rat chow for $5 \mathrm{~min}$ and recording the amount of time that each animal possessed and ate the pellet. The winner of each such encounter was the contestant with the higher score. To ensure that the animals would compete for food, testing was preceded by a training period during which the gerbils were individually placed in the tank for $5 \mathrm{~min}$ daily, and training was concluded only when all Ss ate for a minimum of $200 \mathrm{sec}$ during each of two consecutive daily sessions. This appeared to be effective in that there were very few test sessions in which the food pellet lay unpossessed for more than a few seconds, and competition for the pellet was characteristic of most encounters. Testing was conducted during the midafternoon of consecutive days in a quiet room, with the $\mathrm{E}$ remaining as still as possible while recording. All animals had colored earmarks to facilitate recognition and received their daily food ration, which was as much rat chow as they could consume during a 45 -min period, in their home cage at least $30 \mathrm{~min}$ after testing.

The apparatus used for the competitive avoidance testing was a Scientific Prototype A-100 test cage containing an aluminum shelf, just large enough to support one gerbil, attached to a wall $4 \mathrm{~cm}$ above the floor. The grid floor was attached to a Grason-Stadler E6070B shock generator programmed to emit a 1 -sec $2-\mathrm{mA}$ shock every $15 \mathrm{sec}$. Testing consisted of placing a pair of gerbils in the box and noting which animal occupied the shelf at the onset of each of 20 shock trials, the winner here being the animal who avoided the most shocks. As in food competition, testing was preceded by a training period, during which animals were individually trained to avoid shock by occupying the shelf, and training was conducted until all Ss avoided at least 18 of the 20 shocks on each of 2 consecutive days.

All animals were tested against all other members of their group in a round-robin fashion, twice under food competition and twice under competition for shock avoidance. To counterbalance for the effects of order, one group received the two food tests first, the other the two shock tests. Each test consisted of 66 competitive encounters which were conducted during an 11-day period, with each animal being tested once per day. Food and shock tests were conducted on the same day, with the food trials preceding those for shock. During a 1-day period between Test 1 and Test 2 and between Test 3 and Test 4 (i.e., between the tests for the same incentive), the Ss were individually retested to ascertain if they were still at criterial levels, and, in all cases, they were. Between Tests 2 and 3 , there was a 10-week period during which all animals were trained for competition for the new incentive.

\section{Results}

Analysis for determining the consistency of competitive outcomes can be in terms of intratest regularity or interest reliability. The first kind of analysis is concerned with whether or not there is a dominance hierarchy: Is the distribution of wins and losses other than that which would be expected by chance? Using a modification of chi square (David, 1959), outcomes on three of the four food tests and on three of the four shock tests were significantly hierarchical $(p<.05)$. Only the first tests for each group showed no transitive arrangement of scores, though none of the hierarchies were perfectly linear.

With regard to intertest consistency, the Spearman thos based on the number of encounters won for each animal were .04 and .08 between successive food competition tests, .55 and .29 between successive avoidance tests, and .42 and .23 between the tests for different incentives, none of which are significant at $p<.05$. Analysis in terms of consistency of paired outcomes across the four tests (i.e., is the winner in a given trial likely to win the next time he competes against the same animal?) by the binomial test indicated reliable consistency $(p<.05)$ only between successive avoidance tasks.

Since dominance relationships are facilitated by mutual recognition, animals housed together should show greater intertest consistency than those housed separately. Comparisons of percentage of similar outcomes between successive tests were made for cagemates and noncagemates using chi square, and these indicated no reliable differences.

To ascertain the role of nonsocial factors in performance in the competition tests, the correlations between performance during the criterial training trials and number of contests won in the immediately succeeding test were calculated. The Spearman rhos for the two groups were .27 and .56 for the food incentive and .60 and .46 for shock avoidance. These coefficients probably greatly underestimate the magnitude of the relationship between the variables since the 
range of scores on the criterial trials was necessarily a narrow one-between 200 and $300 \mathrm{sec}$ on the food trials and 18 and 20 avoidances on the shock trials. Nonetheless, the two highest coefficients were significant at $\mathrm{p}<.05$. These results, along with the failure to find significant consistency across the different incentives, as well as the absence of any observable displays correlated with either winning or losing, suggested that the obtained intratest consistency could probably be better explained by individual differences in skill at obtaining incentives than by the concept of social dominance.

\section{EXPERIMENT 2}

Though the results of Experiment 1 were largely negative with regard to the existence of dominance relationships in gerbils, an additional test was made. Taking the view that such relationships exist to regulate competition and consequent aggression, it was hypothesized that intensification of competition by increasing the level of motivation of the participants would result in competitive outcomes that were more hierarchically arranged and more stable over time. Methods

The Ss were 32 adult male gerbils obtained from Tumblebrook Farms, Brant Lake, New York, randomly divided into four groups of eight each and housed four to a cage. The same shock avoidance apparatus and training, testing, and scoring procedures were used. Each group was assigned to one of four shock values: .5 , $1.0,2.0$, and $4.0 \mathrm{~mA}$. The use of $.5 \mathrm{~mA}$ was determined on the basis of a pretest as the lowest in a series of intensities which reliably resulted in a detectable response; the $4.0-\mathrm{mA}$ value was one which regularly gave highly excited jumping and squealing without mortality or visible burns. The animals were trained and tested under the same intensity, and testing consisted of three round-robins in each of which every animal competed once against the animals in his group. Each test was conducted during 7 successive days, with four different pairs of animals from each group tested every day. The tests were separated by a 16-day period, on the last 3 of which the animals were retrained to criterion.

$$
\text { Results }
$$

The chi squares for intratest consistency appear in Table 1. As may be seen, the arrangements of wins and losses were significantly hierarchical in 9 of 12 cases; however, only in Test 3 was there any apparent relationship between shock intensity and consistency of competitive outcome. Even this suggestion of a
Table 1

Chi Square for Intratest Consistency (Hierarchy)

\begin{tabular}{lllc}
$\begin{array}{l}\text { Shock } \\
(\mathrm{mA})\end{array}$ & Test 1 & Test 2 & Test 3 \\
\hline .5 & $19.25^{* *}$ & $14.25^{*}$ & 9.25 \\
1.0 & 11.00 & $19.00^{* *}$ & 12.00 \\
2.0 & $14.00^{*}$ & $16.00^{*}$ & $15.00^{*}$ \\
4.0 & $17.50^{*}$ & $17.50^{*}$ & $21.00^{* *}$ \\
\hline${ }^{*}<.05$ & ${ }^{* *} p<.01$ &
\end{tabular}

relationship was vitiated by analysis of differences in extent of polarization of scores, in which the percentages of trials in each contest won by the winner were compared for the different conditions. No orderly relationship was found between shock intensity and degree of polarity on any test, nor were there any reliable differences in polarity across tests. Analysis for intertest consistency indicated that the Spearman thos for number of contests won between Test 1 and Test 2 were significant at $\mathrm{p}<.05$ for the groups tested under .5 , 1.0 , and $4.0 \mathrm{~mA}$ and that the rhos between Tests 2 and 3 were significant for all groups. These rhos ranged between .49 and .85 and showed no apparent relationship to shock intensity. A similar analysis of the percentage of identical outcomes across tests revealed the same consistency unrelated to shock levels.

Other analyses showed no apparent relationship between the weight of the animals and success in competition for any group on any test, or any differences in consistency of outcomes when the scores of cagemate pairs were compared to those of noncagemates. As in Experiment 1, there was an overall positive relationship between performance during the criterial training days preceding the tests and success in competition. These data are presented in Table 2.

\section{DISCUSSION AND CONCLUSIONS}

The presence of significant intertest reliability when the same incentives were used and its absence across competition for different incentives involving different motor patterns is in agreement with the recent work of Boice, Hughes, \& Cobb (1969), who tested gerbils in competition

Table 2

Spearman rhos Between Performance During Criterial Training Days and Subsequent Number of Wins

\begin{tabular}{llll}
\hline $\begin{array}{l}\text { Shock } \\
(\mathrm{mA})\end{array}$ & Test 1 & Test 2 & Test 3 \\
\hline .5 & $.98^{* *}$ & $.86^{* *}$ & .40 \\
1.0 & .34 & .25 & $.64^{*}$ \\
2.0 & .51 & $.89^{* *}$ & -.13 \\
4.0 & .40 & .57 & .14 \\
\hline${ }_{p}<.05$ & ${ }^{* *} p<.01$ &
\end{tabular}

for food and water. In that study, as in the present ones, no displays correlated with either winning or losing were noted. Since dominance relationships, in order to be functional in regulating competition, must be based on easily recognized displays so that mutual social status may be determined and fighting thereby inhibited, and since such regulation would presumably extend to more than one competitive situation, it is highly unlikely that dominance is operative in gerbils. The failure to find any such displays, as well as any evidence of greater consistency in competitive outcomes under the more intense conditions of Experiment 2, supports such a conclusion. The consistency within tests and between tests for the same incentive found in both experiments can be explained in terms of individual differences in the level of skill required for each task, as evidenced by the preponderantly positive correlations between training and test scores. It is quite possible that reports of dominance in other rodents, based on tests for a single incentive and citing no displays correlated with victory or defeat (e.g., Baenninger, 1966; Becker \& Flaherty, 1968; Lindzey, Thiessen, Blum, \& Tucker, 1969), may be similarly explained.

This leaves unanswered the question as to what does inhibit aggression in gerbils. Other possibilities, such as the presence of specific olfactory stimuli, are currently being investigated.

\section{REFERENCES}

BAENNINGER, L. P. The reliability of dominance orders in rats. Animal Behaviour, 1966, 14, 367-371.

BECKER, G., \& FLAHERTY, T. B. Group size as a determinant of dominance-hierarchy stability in the rat. Journal of Comparative \& Physiological Psychology, 1968, 66, 473-476. BOICE, R., HUGHES, D., \& COBB, C. J. Social dominance in gerbils and hamsters. Psychonomic Science, 1969, 16, 127-128.

DAVID, H. A. Tournaments and paired comparisons. Biometrika, 1959, 46, 139-149. LINDZEY, G., THIESSEN, D. D., BLUM, S., \& TUCKER, A. Further observations on social dominance in mice. Psychonomic Science, $1969,14,245-246$.

THIESSEN, D. D. The roots of territorial marking in the Mongolian gerbil: A problem of species-common topography. Behavior Research Methods \& Instrumentation, 1968, 1, 70-76.

WYNNE-EDWARDS, V. C. Animal dispersion in relation to social behavior. New York: Hafner, 1962.

\section{NOTE}

1. Naumann, D. J. Statement of preliminary investigation and observation of the Mongolian gerbil Meriones unguiculatus. Unpublished manuscript, 1963. 